\title{
Students' Experiences of Interprofessional Supervision: Shared Characteristics of the Caring Professions
}

\begin{abstract}
The literature on interprofessional supervision suggests that it is possible to supervise some skills but not discipline specific ones. As a result, students' scopes of practice are often limited when supervisors do not have the necessary expertise to supervise them, and such interprofessional placements may be regarded as less than optima learning environments. The aim of this study was to evaluate students' perceptions of the effectiveness of an interprofessional placement for developing their practice capabilities. This paper presents a case study of an interprofessional placement in Australia where complementary medicine students were supervised by nurses and allied health practitioners in mainstream health facilities. Twenty-one students recorded their experiences of this placement in reflective journals. These journals were thematically analysed using an iterative process of constant comparison.

Key themes that emerged from students' reflections on their interprofessional placement suggested that students were developing four core practice capabilities: person-centered care, effective communication, multidisciplinary care, and professionalism. Rather than being a poor substitute for a placement where students are supervised by practitioners from their own profession, this placement was valuable in its own right through its capacity to demonstrate the many practice capabilities that professions have in common. Interprofessional placements could influence shaping students identity, either as a member of a multidisciplinary team or as an outsider.
\end{abstract}

\section{Introduction}

New models of interprofessional clinical placements are being trailed in a range of health disciplines. Drivers for these models are looming workforce shortages and the need to cultivate safe, personcentered, co-ordinated and responsive health care [1]. It has been argued that interprofessional clinical placements may require a different kind of supervision and therefore specific training to clarify roles and to develop core skills of supervision and assessment in an interprofessional environment [2]. The literature on interprofessional supervision suggests that it is possible to supervise some skills but not discipline-specific ones [3]. As a result, students' scopes of practice are often limited when supervisors do not have the necessary expertise to supervise them, and such interprofessional placements may be regarded as less than optimal learning environments. In this paper we argue that placements using interprofessional supervision, rather than being a poor substitute for placements where students are supervised by practitioners from their own profession, are valuable in their own right through their capacity to demonstrate the many practice capabilities that professions have in common. This paper presents a case study of an interprofessional placement where complementary medicine students were supervised by nurses and allied health practitioners.

\section{Journal of} Integrative Medicine \& Therapy

Sandra Grace* and Annette Morgan

School of Health and Human Sciences, Southern Cross University, Australia

*Address for Correspondence

Sandra Grace, School of Health and Human Sciences, Southern Cross University, PO Box 157, Lismore, NSW 2480 Australia, Tel: 612-66269258; E-mail: sandra.grace@scu.edu.au

Copyright: (c) 2015 Grace S, et al. This is an open access article distributed under the Creative Commons Attribution License, which permits unrestricted use, distribution, and reproduction in any medium, provided the original work is properly cited.

Submission: 11 February 2015

Accepted: 24 February 2015

Published: 02 March 2015

Reviewed \& Approved by: Dr. Adam Perlman, Executive Director, Duke Integrative Medicine, Durham

Interprofessional practice refers to collaborations and networks among practitioners of different disciplines, including complementary medicine practitioners. Over $50 \%$ of the Australian population utilize complementary medicine $[4,5]$, which may present a problem for health practitioners who do not have a sufficient level of understanding or expertise to advise their patients about its use [6,7]. The case for complementary medicine education for both students and practitioners of other health disciplines is well documented, particularly in pharmacy, medicine, nursing and physiotherapy [811]. Interprofessional placements in pre-professional education can provide fertile opportunities for students to work with, and learn about, the contributions of diverse approaches to their patients' care, and provide vital preparation for future collaborative practices. However, there appears to be few opportunities for interprofessional placements that accommodate mainstream and complementary medicine students.

In Australia, osteopaths are non-medical practitioners who work primarily in private practices in the community. An innovative placement was established with students from Southern Cross University's osteopathy program in 2009. In this placement students work in palliative care and rehabilitation wards of local hospitals and in aged care facilities. These are the only placements in Australia where student osteopaths work in mainstream health facilities. They are supervised by health professionals from disciplines other than their own, usually nursing professionals. Students also observe nurses, allied health professionals and medical practitioners at work. The intended learning outcomes of these placements are not to develop discipline-specific procedural skills, but practice capabilities like communication skills with patients and other health care professionals, teamwork, and critical self-reflection. These capabilities are those expected in university graduates in all health disciplines and have been documented among medical students following clinical placement experience [12]. The aim of this study was to evaluate students' perceptions of the effectiveness of an interprofessional placement for developing their osteopathic practice capabilities. 


\section{Methods}

The study is informed by Brookfield's [13] theory of critical selfreflection and draws on written reflections of osteopathic students in mainstream medical hospital placements. Clark [14] identified journal writing as an important tool for learning and improving performance, although it appears that some students benefit more than others from the activity [15]. Journal writing can serve many purposes in a course including self-expression, a record of events, a form of therapy, or an avenue for critical self-reflection (as in this study), and is influenced by the conditions under which it occurs (e.g. assessed or not assessed) [16]. Hospital placements provide students with a very different work environment from those in which osteopathic students usually train and those in which osteopaths in Australia typically practice. In such a novel work environment, critical self-reflection can prompt students to identify their emotions and assumptions, consider the social and personal contexts which go to form them, and imagine alternative ways of thinking and behaving $[14,17]$.

A case study design was used to focus on a cohort of third year osteopathic students undertaking 24 hours of clinical placement over a four-week period in either of two regional hospitals' palliative care or rehabilitation wards, or in local aged care centres. Case studies can be used to understand phenomena in complex social situations and to formulate hypotheses for future research [18]. In this study, the case under investigation was an interprofessional clinical placement where students from a complementary medicine discipline were located in either a mainstream medical hospital or a community aged care facility. The case study research proposition was that health students who are supervised by a supervisor from a discipline other than their own can develop capabilities that are essential for practice. The research questions which followed from this proposition included:

- From students' perspectives, what capabilities can be developed on interprofessional placements?

- From students' perspectives, does interprofessional supervision limit their ability to develop core practice capabilities?

The students were given guidance about how to complete their reflective journals. They were encouraged to write at the end of each clinic shift and advised that the journals did not form part of the assessments for the placement. Invitations were sent via email to the third year cohort after all placements had been completed and 21 students agreed to participate. With students' consent, reflective journals were de-identified and analysed by two researchers, each of whom repeatedly read and re-read students' written reflections in order to code initial concepts. The two researchers then met and through a process of constant comparison [19] clustered, categorised and refined concepts until key themes were identified.

\section{Results}

Reflective journals documented students' observations of patients' progress, responses to treatment, interactions with hospital staff, hospital staff interactions with each other, and the students' own interactions with both these groups. Journals also documented students' critical self-reflections on their experiences. Key themes emerging from student reflections were:

\section{Embodied illness}

Students encountered patients with conditions that they knew only from their lectures and readings. Placement in hospitals allowed them to experience a number of medical conditions and disease as they were embodied in patients. This first experience in the mainstream health system focused their attention firmly on patients and their illness experiences.

Today for the first time I fully understood the term patient individuality. Before today I thought I knew what this term meant but failed to physically comprehend this. Today I witnessed the difference between two male patients both of whom are undertaking rehabilitation of the same condition but each one expresses this condition to me in vastly different ways. (Geoff)

First day was quite confronting. It was eye-opening to see the emotion and suffering that patients who have the conditions that we study at university actually experience. A stroke patient spoke of being fit and healthy and one day when he was on his daily $7 \mathrm{~km}$ walk with his dog the stroke occurred and he is now having to learn to walk, talk and regain arm movement. I saw a man with Parkinson's break down in tears telling me how debilitating it is and how he had seven falls in seven days. I don't feel that much can really prepare you for experiences like that. (Ellen)

\section{Communicating with patients}

Learning to communicate with patients was challenging for some students. They had to overcome feelings of awkwardness and shyness in a new environment and a new role.

What I noticed from my first day was my difficulty talking to patients about their illnesses. I am now finding this to be a stupid thing as all the patients I have come across are understanding and once they know a little about me and why I am here they are more than happy to share their stories. (Tim)

Today I was surprised at how grumpy and hostile patients in pain can be. It was frustrating that even though I am here volunteering I was not being treated with any gratitude. It was upsetting. I can understand that the patient may have been in a great deal of pain, had a bad day or maybe they think I am a paid staff member. I found all this challenging but I am glad to have experienced this now because now I'll be more prepared to deal with the situation. (Geoff)

\section{Collaborative care}

For these third year students, the hospital or aged care centers were their first encounters, as health professionals, with multidisciplinary team care.

We were invited to attend one of the doctors' conference meetings which involved the physios, OTs, speech therapists and some nurses and doctors to review patients' progress. The conference was a great experience to see how different professions all work together to maximize the benefits of the patients' stay; try to help them with preparing their homes for their return, and assessing progress in their various fields. It was good to see the inter-practitioner communication and the teamwork involved in running the rehab centre and getting patients home again. (Trish) 
They could see the potential for their inclusion in such teams and they were learning about their place as students, and as students of osteopathy in the health care system.

I looked through a number of patient files to see the variety of different problems that the patients are in there for. I was interested in seeing how the physiotherapists work on each patient as well as the OTs so I read the notes they recorded in the files. It was pleasing to read notes on neuro, cardio, respiratory, GIT, renal and psychology and be able to understand them from what we've learned this year at university. (Ellen)

I managed to have a chat with one of the nurses ... I was rather surprised to see how much she actually knew about osteopathy and was a big believer in the work we do. It was nice to hear that coming from someone from a different discipline. (Grant)

A hierarchical system based on professions was evident to some students.

I felt that my responsibilities toward the patient were small compared to those of doctors, surgeons or physios. Nevertheless I believe that our responsibilities will increase with our learning ... Sometimes I find it a bit frustrating to be regarded as less than the physios. I believe that osteopaths can do a great deal but otherwise I enjoyed the placement. (Phil)

\section{Developing professional identity}

This placement provided many students' first experiences as 'practitioners' beyond the simulations in practical classes. They were beginning to understand the clinical responses to their soft tissue techniques. They had a large emotional investment in patients' responses to their treatment and reported moments of elation and moments of dejection, depending on patients' responses to their treatments. The following extracts reveal these students taking on their 'professional skin'. There were significantly more reflections about this theme than any other.

Today is the last day in the rehabilitation centre before the 73 year old male CVA patient is discharged. It was such an incredible experience to be able to see him every time I had a shift and really get to observe his progress. To see the achievements however small they may seem meant so much to me. I saw his fingers move again for the first time when I was treating him, watched his movement improve every time I saw him. I am just so grateful to have had the opportunity to come to a place where they are more than happy to have students. It has taught me so much and the feeling you get when you see that the patients can go home is so satisfying. He is the second one of the patients I treated that has been discharged and they have both thanked me immensely and it just makes you feel invaluable. (Natalie)

It is a nice feeling to know that what I am doing is making some difference even if it is just giving them a morale boost by having someone different to talk with who has yet to hear their story. (Geoff)

His arm was beginning to resemble the opposite side. The most exciting improvement from my point of view was the random twitching movements of their $2^{\text {nd }}$ digit left side, the affected side. To see these improvements has given me joy to witness how beneficial the work is here. I also like to think that I am playing a small role in helping the body in its recovery process. (John)

\section{Discussion}

Using journal writing enhanced students' learning in a number of ways: they deepened their understanding of patients' experiences of illness and suffering; they reflected on the significance of being in a caring profession and the influence they can have on patients' experiences; and they reflected on their profession's role in collaborative care. In some journals, there was evidence of transformation by first-hand experiences with patients' debilitation and suffering. Students were elated with their patients' recoveries. They were transformed by the realization that they could contribute to those recoveries, albeit perhaps in small ways, such as providing a 'morale boost', as one student put it. This is the transformation referred to by Clouder [20] when talking about 'caring' as a threshold concept that transforms students into health professionals. However, students in this case study did not reveal difficulties in taking on caring responsibilities, such as might have been expected in coming to terms with the 'troublesome knowledge' [21] that such encounters could present. Despite the potential emotional challenge of not only observing the signs and symptoms of illness but also engaging with its lived experience, students' reflective journals invariably reported positive learning experiences.

The aim of this study was to explore the development of professional capabilities as revealed through students' reflections. The four key themes of this study can be categorized as the following capabilities: (1) 'embodied illness' or understanding patients' illness experiences as part of the capability of patient-centered care; (2) 'communicating with patients' as part of the capability of effective communication; (3)'collaborative care' as part of the capability of multidisciplinary care; and (4) 'developing professional identity' or learning to embrace the culture of the profession as part of the capability of professionalism - all of which are core capabilities of all health professions [22,23]. Competency frameworks clearly articulate scopes of practice and enable professional boundaries to be set. However, as Reeves et al. [24] point out, the 'competency movement ... limits innovation and interferes with interprofessional practice' (p451). In this interprofessional placement students had the opportunity to assume the generalized culture of the health professions, or the specific identity of an osteopath.

A further aim of the study was to investigate whether students' felt that interprofessional supervision had limited their ability to develop their osteopathic practice capabilities. The capabilities identified above (patient-centered care, effective communication, multidisciplinary care and professionalism) are generic ones - a student from any health profession could have developed these skills in a similar learning environment. The question is whether the students in this study could have further developed their osteopathicspecific skills under supervision of practitioners from other disciplines. The selection of assessment and treatment techniques that were deemed safe by supervisors and students to practice on these specific placements were all within the scope of osteopathic practice. Clearly patient safety would be at risk should students practice techniques that could be harmful if applied inappropriately, and from that perspective, a supervisor from a discipline other than osteopathy 
Citation: Grace S, Morgan A. Students' Experiences of Interprofessional Supervision: Shared Characteristics of the Caring Professions. J Integrative Med Ther. 2015;2(1): 4.

could limit the range of techniques that osteopathic students could safely apply. However, in such settings as rehabilitation hospitals and aged care facilities, the appropriate osteopathic techniques are the gentle ones that can be safely applied under the supervision of any health practitioner. An osteopathic student supervised by an osteopathic supervisor would perform similar treatment techniques in these learning environments. The interprofessional supervision in this study, rather than being an inferior substitute for samediscipline supervision, provided a unique opportunity for students to understand many generic aspects of the caring professions. They developed communication and teamwork skills; emotional maturity through person-centered encounters; and they began to assume their professional identity. Interprofessional cultures could influence shaping that identity, as either different from, or the same as, those of other disciplines, and as marginalized or belonging to the health care team.

This case study is context-dependent and the findings are not intended to be generalised. Findings may be useful for academics and supervisors who are designing interprofessional placements with cross-disciplinary supervision. They may also be useful for academics and supervisors who are considering their students' use of critical self-reflection in their journals, including reflections on their emotional responses to patients and treatments, on workplace cultures, and on their own assumptions and preconceptions. In future research we need also to understand the experiences of supervisors of students from different disciplines, particularly from the perspective of marginalization and belonging in the workplace and of the relative merits of generic and discipline-specific capabilities in preparing the future health workforce.

\section{Conclusion}

In an interprofessional placement where osteopathic students were supervised by nursing and other health care professionals, students' journal entries recorded observations of patients' progress, their interactions with hospital staff and patients, and their emotional responses to their experiences. Journal entries also revealed critical self-reflections on those experiences. Key themes emerging from student reflections suggested that students were developing four core practice capabilities: person-centered care, effective communication, multidisciplinary care, and professionalism. Interprofessional placements could influence shaping students' identity, either as a member of a multidisciplinary team or as an outsider. Rather than being an inferior substitute for same-discipline supervision placements, this study suggests that placements where students are supervised by practitioners from another discipline can provide a unique opportunity for students to understand the shared characteristics of the caring professions.

\section{References}

1. Health Workforce Australia (2012) National common health capability resource: Shared activities and behaviours in the Australian health workforce. Adelaide.

2. Thistlethwaite JE (2013) Practice-based Learning Across and between the health professions: A conceptual exploration of definitions and diversity and their impact on interprofessional education. Int J Practice-based Learn Health Soc Care 1: 15-28.

3. Chipchase L, Allen S, Eley D, McAllister L, Strong J (2012) Interprofessiona supervision in an intercultural context: A qualitative study. J Interprof Care 26: $465-471$.

4. MacLennan AH, Myers SP, Taylor AW (2006) The continuing use of complementary and alternative medicine in South Australia: costs and beliefs in 2004. Med J Aust 184: 27-31.

5. Xue C, Zhang A, Lin V, Da Costa C, Story D (2007) Complementary and alternative medicine use in Australia: A national population-based survey. $\mathrm{J}$ Altern Complement Med 136: 643-650.

6. Gaylord SA, Mann JD (2007) Rationales for CAM education in health professions training programs. Acad Med 82: 927-933.

7. Lee A, Foong Y, Le H (2012) Complementary and alternaive medicine and medical students in Australia: Where do we stand? Australa Medl J 5: 144149.

8. Laurenson M, MacDonald J, McCready T, Stimpson A (2006) Student nurses' knowledge and attitudes toward CAM therapies. Br J Nurs 15: 612615.

9. Nedrow A, Istvan J, Haas M, Barrett R, Salveson C, et al. (2007) Implications for education in complementary and alternative medicine: A survey of entry attitudes in students at five health professional schools. J Altern Complement Med 13: 381-386.

10. Bjersa K, Sterner Victorin E, Fagevik Olsen M (2012) Knowledge about complementary, alternative and integrative medicine (CAM) among registered health care providers in Swedish surgical care: A national survey among university hospitals. BMC Complement Altern Med 12: 42.

11. Tiralongo E, Wallis M (2008) Attitudes and perceptions of Australian pharmacy students towards Complementary and Alternative Medicine - a pilot study. BMC Complement Altern Med 8: 2 .

12. McNeil HP, Scicluna HA, Boyle P, Grimm MC, Gibson KA, et al. (2012) Successful development of generic capabilities in an undergraduate medical education program. High Educ Res Dev 31: 525-539.

13. Brookfield S (1995) Becoming a Critically Reflective Teacher. WILEY.

14. Clark P (2009) Reflection on reflection in interprofessional education. J Interprof Care 23: 213-223.

15. Chirema K (2007) The use of reflective journals in the promotion of reflection and learning in post-registration nursing students. Nurse Educ Today 27: 192-202.

16. Boud D (2002) Using journal writing to enhance reflective practice. New Directions for Adult and Continuing Education 2001: 9-18.

17. Parrish D, Crookes K (2014) Designing and implementing reflective practice programs - Key principles and considerations. Nurse Educ Pract 14: 265270.

18. Yin RK (2003) Case Study Research. Design and Methods. 3rd edition, Thousand Oaks Ca: Sage.

19. Glaser BG, Strauss AL (1967) The Discovery of Grounded Theory: Strategies for Qualitative Research. Chicago, Aldine Publishing.

20. Clouder $L$ (2005) Caring as a threshold concept: transforming students in higher education into health (care) professionals. Teach High Educ 10: 505 $-517$

21. Meyer JHF, Land R (2003) Threshold concepts and troublesome knowledge: linkages to ways of thinking and practicing. In: Rust C, editor. Improving student learning - Theory and Practice Ten Years On. Oxford: Oxford Centre for Staff and Learning Development 412-424.

22. Verma S, Paterson M, Medves J (2006) Core competencies for health care professionals: What medicine, nursing, occupational therapy, and physiotherapy share. J Allied Health 35: 109-115.

23. Pruitt S, Epping-Jordan J (2005) Preparing the 21st century global healthcare workforce. Br J Med 330: 637.

24. Reeves S, Fox A, Hodges B (2009) The competency movement in the health professions: Ensuring consistent standards or reproducing conventional domains of practice? Adv Health Sci Educ 14: 451-453. 